\title{
COVID-19 related factors affecting the experiences of neurodivergent persons in the workplace: A rapid review
}

\author{
Damian Mellifont* \\ Centre for Disability Research and Policy, The University of Sydney, Sydney, NSW, Australia
}

Received 18 May 2021

Accepted 23 August 2021

\begin{abstract}
.
BACKGROUND: Policy responses to the COVID-19 pandemic offer possibilities to advance social justice. One such prospect is to make workplaces more inclusive of neurodivergence.

OBJECTIVE: This research addresses the question of, in what ways might COVID-19 affect the experiences of neurodivergent persons in the workplace?

METHODS: Conducting a rapid review, the author has applied thematic analysis to a total of 50 documents comprised of journal articles, news articles, and guides as retrieved from purposive searches of ProQuest Central, ProQuest Newsstream International, Google Scholar, and Google databases.

RESULTS: Research results have revealed themes of challenges and opportunities, and sub-themes of accommodating (i.e., remote working, employee recruitment, retainment or advancement and/or access); and diversity and inclusion (i.e., acceptance, empathy and/or ERGs).

CONCLUSION: This study has informed a baseline COVID-19-related guide to accommodating and including neurodivergence in the workplace. The review concludes by offering possibilities as to what a COVID-19 inspired 'new normal' might mean for supporting neurodivergent staff (and prospective staff).
\end{abstract}

Keywords: COVID-19, neurodiversity, inclusion, equity, diversity, accommodations

\section{Introduction}

\subsection{Neurodiversity and the neurodiversity movement}

Attributed to Judy Singer (sociologist) in the late 1990s and promoted by the journalist, Harvey Blume, the neurodiversity concept is relatively new $[1,2]$. The term neurodivergent has been defined as, "having

*Address for correspondence: Damian Mellifont, Honorary Postdoctoral Fellow of the Centre for Disability Research and Policy at The University of Sydney, Sydney, Australia. E-mail: damian.mellifont@sydney.edu.au. a brain that functions in ways that diverge significantly from the dominant societal standards of "normal"' [3]. This contrasts against the majority of persons who are neurotypical (i.e., their brains process information in ways that meet the expectations of society) [4]. Neurodiversity references the various ways in which brains function and understand information [4]. It positions neuro types such as dyslexia, autism and Attention Deficit Hyperactivity Disorder (ADHD) as natural differences in cognition [2]. Neurodiversity offers a change of perspective which serves to challenge presumed perceptions of normality [5]. As a label, neurodiversity has been 
described as showing more respect for the dignity of humans [6]. Supporting principles of social justice, this respect should extend to the significant number of neurodivergent persons who are present in a populace. The proportion of neurodivergent citizens is notable. It is suggested that around ten percent of the population has some kind of neurodivergence [7].

Neurodiversity holds a political aspect. A discussion as held among autistic persons and individuals with other kinds of neurodivergence has laid the groundwork for the neurodiversity movement (NDM) [8]. This movement aims to deliver changes in how society looks at and reacts to neurological diversity [9]. The NDM largely involves advancing the civil rights of neuro-minorities [10]. The movement also holds a solid grounding in scientific studies that record the abilities of persons with various medical labels [11]. Donnelly [12] makes the point that some neurodivergent persons are intellectually gifted. Challenging the medical model, Kapp, GillespieLynch, Sherman and Hutman [13] report of the NDM pursuing quality of lived experiences over cure. The movement, however, is not without its critics. There are various disability advocates who are anxious that the movement downplays the challenges confronting individuals with severe cognitive issues [1]. Rothstein (14, p.107) suggests it to be "cruel" to assign the neurodiversity term to those persons who require intensive medical treatments. Russell [15] also recognises an allegation that the movement is not representative of all neurodivergent persons. Nonetheless, the potential for the NDM to support progressive and prideful meanings about neurodivergence should not be downplayed or dismissed.

\subsection{Neurodivergence and career development barriers}

A greater understanding is required of the issues that can damage the careers of neurodivergent persons. Davila, Shectman and Anandan [16] suggest that a need exists to more deeply address the area of inclusion in the workplace, and in particular in relation to the participation of neurodivergent employees. Such inclusion can be undermined by inflexible and inappropriate assessment measures. In this sense, interviews are prejudiced against persons who are atypical in their interactions [17].

Workplaces can also overlook the abilities and requirements of neurodivergent employees $[1,16]$. These oversights are not adequately redressed through current policy. It has been reported that nearly 75 percent of workplace policies disregard neurodivergence [7]. The consequences of this policy shortfall can be serious. For instance, open plan office designs are "distracting and unproductive" for neurodivergent employees who are sensitive to sound (18, p.5). Neurodivergence is also reported as being overrepresented among persons who are experiencing long-term unemployment [19]. Contemporary workplaces are challenged to recognise and redress these kinds of persisting inequities.

\subsection{COVID-19 widespread devastation and the careers of neurodivergent persons}

COVID-19 (forthwith to be referred to as COVID) represents a perplexing policy issue. Over a very short space of time, life has been interrupted on a global scale [20]. With 27 cases identified in Wuhan City, China in December 2019, and having not before been detected among humans, COVID-19 is now referred to as a "devastating pandemic" (21, p.S3, 22, p.543, 23). COVID-19 has been depicted as an unparalleled test for healthcare [24]. For numerous persons around the world, the pandemic has shown itself to be the difference between life and death. In this light, COVID-19 is ranked as a prominent cause of fatality [25]. Despite improvements in treatments and promising vaccines, COVID-19 is likely to continue to be around for many years [26].

COVID-19 can threaten not only physical health, but also mental wellbeing. Thite $(27, \mathrm{p} .13)$ describes the pandemic as unleashing "chaos and uncertainty". This global pandemic has disrupted daily habits, seen a revival of xenophobia, and wounded the economy [20]. Prolonged time spent in isolation together with the stressors of dealing with COVID-19 have tested the mental health of many persons [28]. Davila, Shectman and Anandan [16] recognise that an environment has been generated whereby anxiety and stress is elevated for everyone. Donnelly [12] cautions that COVID-19's interruption of work and home habits has proven to be a significant event for many employees. The initial wave of jobs that have been lost during COVID-19 has been immense [29]. The pandemic has thus generated intense fear and doubt across personal and working lives [30].

As the world struggles with COVID, each day brings with it additional headlines concerning the future of the workplace [1]. While the workplace can be a perplexing and possibly frightening environment for some neurodivergent persons in usual times, it will likely become even more overpowering due to 
COVID-19 driven uncertainty [31]. Nonetheless, the pandemic is encouraging employers to adjust [32]. COVID-19 is starting to modify views throughout society with many having gained a keener awareness of their environments [2, 33]. Possibilities for COVID-19 disruptions to influence the careers of neurodivergent persons are worthy of scholarly attention. Hence, this rapid review will address the timely question of, in what ways might COVID-19 affect the experiences of neurodivergent persons in the workplace?

\section{Method}

The steps taken to conduct this rapid review were informed by an approach as indicated by Khangura, Konnyu, Cushman, Grimshaw and Moher [34] in their comparison of this type of review to a systematic review. This approach involved the following six steps: 1) setting a research question; 2) identifying the sources of data; 3 ) developing a search strategy; 4) selecting data by applying criteria; 5) synthesising the data; and 6) cautiously interpreting the findings.

\subsection{Qualitative data collection}

A research question (as stated above) was firstly set. Next, the following databases with capacity to inform this review were selected: ProQuest Central; ProQuest Newsstream International databases; Google Scholar; and Google (internet enquiry). Being purposefully constructed to inform the research question, the search term was comprised of: COVID-19 AND neurodiver* AND workplace. Inclusion criteria were set as follows: search fields $=$ all fields AND Document type $=$ journal article OR grey literature (i.e., news article OR guide OR report $)$ AND language $=$ English AND publication date $=2020$ to 2021 AND document refers to possible COVID-related factors affecting neurodivergent staff or prospective staff AND document is accessible. Searches were then conducted and document details imported into EndNote X7.8 with duplicates removed. Following on, abstracts, where available, were inspected for possible relevance. Next, possibly relevant documents were accessed and appraised in detail. Scholarly citations from relevant articles were then included in the study results.

\subsection{Qualitative data analysis}

Enabling a synthesis of the data, the author has applied the thematic analysis technique. As informed by Braun and Clarke [35], this analysis involved a repeated cycle of: 1) inspecting the relevant documents; 2) building a coding scheme, and 3) identifying, naming and reviewing themes and subthemes.

\section{Results}

Database search results are provided in Table 1. These results incorporate the database that was queried, the number of documents that were duplicated in the searches, a recalculated number of possibly relevant documents, and the number of relevant documents. Supplementary Table 1 provides details about each of the relevant documents. These details include: the document reference; country of origin; document type, i.e., journal article, news article or guide; document theme(s): challenges; opportunities, and sub-theme(s): accommodating (i.e., remote working, employee recruitment, retainment or advancement and/or access); and diversity and inclusion (i.e., acceptance, empathy and/or ERGs); together with a description of the possible COVID-related opportunities and/or challenges for neurodivergent persons in the workplace. Advancing data transparency, Supplementary Table 2 explicitly expresses the coding rules for each of the themes and sub-themes. Figure 1 provides a hierarchy list chart to graphically represent the themes and sub-themes as revealed through the thematic analysis.

Table 1

Database enquiry results

\begin{tabular}{lcccc}
\hline Database & Possibly relevant & Duplicates & Revised possible & Relevant \\
\hline ProQuest Central & 143 & 0 & 143 & 28 \\
ProQuest Newsstream & 34 & 30 & 4 & 1 \\
Google Scholar & 73 & 2 & 71 & 8 \\
Google & First 4 pages & 0 & $\mathrm{Na}$ & 13 \\
Total & & & & 50 \\
\hline
\end{tabular}




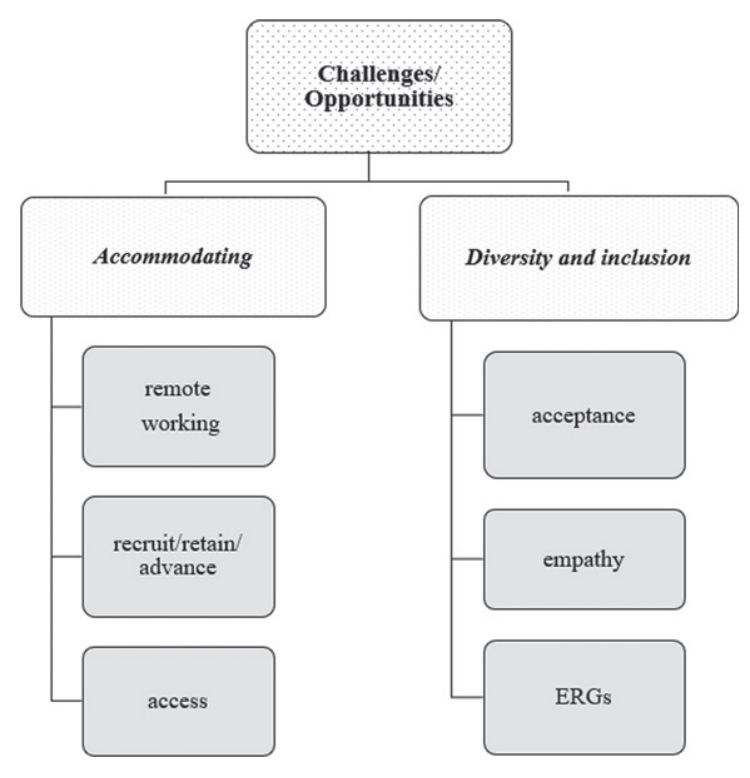

Fig. 1. Themes and sub-themes hierarchy list chart.

\section{Discussion}

The author will critically discuss the COVIDrelated challenges and opportunities that surround: a) accommodating neurodivergent employees; and b) advancing diversity and inclusion (and in particular, the inclusion of neurodivergence) in the workplace. From a practical perspective, this critical discussion will then inform a baseline guide to accommodating and including neurodivergent staff, and prospective staff, both during and post-COVID. Promoting transparency throughout this critical discussion, the author will delineate between those instances where content is informed by scholarly (i.e., peer reviewed articles) and grey literature (i.e., news articles, reports, guides).

\subsection{COVID-related challenges and opportunities for accommodating neurodivergence in the workplace}

Accompanying the pandemic are obstacles and opportunities to accommodating neurodivergence in the workplace. Prominent among COVID-related adjustments is the requirement for staff (including neurodivergent staff) to work from home. Sargent [36] observes in a news article that an enormous, unexpected experiment in remote working is presently underway. Significant concerns, however, are mentioned in the literature about neurodiver- gent employees in this remote work environment. As reported among scholarly and grey literature, such concerns include: micromanagement; video conferencing issues (e.g., fatigue from chaotic Zoom meetings); uncertainty as to the ongoing availability of virtual accommodations; struggles with physical distancing; mental health and wellbeing issues following a disruption to routines; overworking in the home environment; and sensory overload upon returning to the office (e.g., worsening symptoms for OCD employees) [26, 28, 37-46].

Elaborating on Zoom fatigue, it needs to be recognised that not all neurodivergent persons will be comfortable with these kinds of virtual meetings. According to a news report, some might be overwhelmed by many persons who are talking at the same time [45]. The scholarly and grey literature reveal a range of options for those who are uncomfortable with noisy online meetings or with real time, face-to-face interactions. These options include: oneon-one or smaller group Zoom sessions; enabling Zoom captions; reducing anxiety by sending out the agenda beforehand; using chat tools instead of live discussions to raise and address questions; or allowing employees to provide their meeting contributions in advance [16, 45]. News reporting suggests that dyslexic employees might also benefit from making the recordings of Zoom meetings available [45]. Moore [47] too reports of some neurodivergent persons much preferring the precision of text-based communications using tools such as Workplace Chat and Slack.

It should not be assumed that neurodivergentrelated accommodations that are effective in an onsite workplace will always be helpful when applied remotely (e.g., in a home office setting). A University of Sheffield guide supports the testing of pre-COVID19 accommodations for neurodivergent staff in terms of their effectiveness in the home work environment [4]. Workplaces therefore need to invest in independent audits so that an assumption is not made that onsite, approved accommodations for neurodivergent employees will necessarily work well when implemented offsite. This highlights the position that rather than being static, accommodating neurodivergence during a pandemic is a dynamic process.

Reported challenges for neurodivergent staff who are working remotely confront a number of misconceptions about neurodivergence. News articles endorse the notion that autistic employees do not want social contact is a falsehood as these persons can be negatively impacted by the loss of connections that 
are developed over time [1,26]. The ableist notion that neurodivergent staff must be micromanaged is undermined by the Neilson [28] news reporting of some autistic persons overworking (i.e., not taking rest breaks, and missing meals when working from home). The concerns of some managers about the day-to-day productivity of persons who are working from home [37] might therefore be misplaced when these are automatically directed at neurodivergent staff. Still, any suggestion that a neurodivergent person will necessarily thrive in a remote working role is questioned by the Donnelly [12] assertion that some of these persons might struggle when the routines in which they often flourish are disrupted. Testing the validity of such news-based assertions, future research is needed to ascertain the possible degree and ease to which neurodivergent persons might establish new routines when working from home.

Notwithstanding the above-mentioned challenges, scholarly and grey literature indicate that a COVID-19 requirement to work remotely can offer opportunities for neurodivergent staff and their employers. These include: offering communication alternatives (e.g., allowing remote access to all meetings); removing on-site challenges for employees with ADHD (e.g., avoiding busy and distracting office settings); enhancing work on a digital platform; and achieving sustained productivity $[6,16$, $39,48,49]$. Remaining cognisant of the potential for Zoom fatigue [43], Moore [47] nevertheless suggests that some neurodivergent employees might prefer meeting interactions that make use of this technology. News articles also purport that COVID-19 can see some neurodivergent persons being hyperaware about the close physical proximity of other team members, while many can be hypersensitive to physical pressure and subsequently struggle with wearing masks $[26,36]$. For these individuals, remote working via online technologies would most likely offer a welcome relief to these particular sources of 'on-site' workplace stressors. Hence, there are some staff who might perceive a COVID-19 requirement to work from home during lockdowns in terms of a reasonable accommodation for their neurodivergence. Independent audits are again needed to explore the possible extent to which these staff are now successfully working remotely, but who might have had their reasonable accommodation requests to work from home rejected prior to the pandemic.

In their news article, Gilroy and Haque (2, n.p.) note that COVID-19 is changing views throughout society with work from home quickly becoming the "new normal". It is this so-called, new normal, that holds interesting prospects for workplaces to become better at accommodating neurodivergence where needed. Recognising potential work from home benefits including those of increased productivity and reduced stress, decision makers should consider allowing neurodivergent workers who are concerned about returning to their workplaces to continue with remote working wherever their roles allow $[42,50]$. Terise and Armstrong (46, n.p.) depict potential for a pandemic-inspired cultural shift away from obsolete workplace norms as follows:

Employers have an opportunity to use their collective experience of the coronavirus pandemic to drive a positive, cohesive shift in workplace culture. They can redesign the outdated nine-to-five office lifestyle.

According to a news reporting, COVID-inspired adjustments hold implications for the recruitment, retainment and advancement of neurodivergent individuals. Video interviews can be helpful in redressing possible bias among panel members as less emphasis is placed upon first impressions [38]. Gilroy and Haque [2] indicate that the success which has been attained with online interviews means that this option will become more commonplace. Accommodations might also be required around COVID-19 messaging originating from the workplace that some neurodivergent staff might find alarming. For example, an employee who was already obsessively monitoring details concerning the virus was bombarded with unwanted daily COVID-19 updates from their organisation [28]. A reasonable accommodation in this instance would be to curtail the sending of these emails with the objective of maintaining employee safety while not adding to anxiety. Moreover, for those neurodivergent persons who might want to return to the on-site office, accommodating office designs might be required. In this respect, Terise and Armstrong [46] advise of the need to provide quiet area alternatives to open plan office settings.

Schwam [1] cautions that while organisations want to avail accommodations and advertise that they have a neurodivergent workforce, accommodations can involve a lengthy process to implement and sometimes, even reasonable ones can be refused. However, Miller [43] raises the encouraging point that COVID-19 has helped to redress formal excuses for not offering flexible work environments. Managers who automatically refuse accommodations to neurodivergent staff and prospective staff need to be 
held accountable for their discriminatory and unreasonable responses. In this light, ethical workplaces should not tolerate any form of neuro-discrimination.

New reports indicate that pandemic inspired accommodations can also serve to brighten the promotional prospects of neurodivergent employees. Greater use of online communications (as a preferred mode of communicating) can help to raise the profiles of these staff members who might have otherwise been invisible to the managerial raider [2]. This adjustment thus holds potential to assist in developing the careers of neurodivergent employees who might not be at ease with face-to-face interactions. Gilroy and Haque [2] also advise that movement towards platforms that are online means that people have more choices to participate in conversations in ways that they desire. It is important that communications and other COVID-related accommodations that collectively work to support the recruitment, retainment and advancement of neurodivergent persons do not fall out of favour post the pandemic.

News article raise potential for large gatherings, work conferences and social events to be more accessible to neurodivergent persons. In this way, virtual events are suggested to encourage greater participation [38]. For example, as a consequence of COVID, hundreds of healthcare staff have attended virtual sessions of the Pharmaceutical Care Manufacturer Association's annual meeting for 2020 [51]. It is quite reasonable to suggest that at least some of these attendees would be neurodivergent, with a subset of these persons potentially preferring this virtual setting. Beery [39] raises the risk, however, that while academic conferences are currently online, this status quo might change. Similar to the availability of remote meetings and remote work accommodations more broadly, ongoing access to virtual conferences should not be taken for granted.

Scholarly reporting informs that for some autistic employees, virtual interactions can offer respite from the rigors of socialising [44]. A reprieve from social activities, for those neurodivergent persons who might want such rest, is quite different from the removal of access to these activities. It thus remains important that social events remain accessible to neurodivergent employees and that these persons are encouraged to socially engage in ways in which they feel comfortable. In line with news reports, Schwam [1] acknowledges opportunities for social connections to be maintained through such means as virtual social events and newsletter emails. Hence for many persons, the pandemic has helped to make work and social life more accessibility [39]. And while not all neurodivergent employees will need or want to participate in virtual social activities, for those who wish to be involved, access to online social interactions should be availed.

Beery [39] suggests that while persons without disability who are hurrying to resume their faceto-face communications risk reducing accessibility back to pre-COVID-19 levels, opportunities still exist to make accessibility something that is permanent. Such accessible and accommodating workplace environments need to meet the individual needs of neurodivergent staff and prospective staff. According to Terise and Armstrong (46, n.p.), "failing to consult neurodiverse individuals in the design of changes put in place to support them is likely to be ill-informed and could do more harm than good, even if it is well intentioned."

Accommodating neurodivergence during COVID19 (or at any other time) raises the topic of disclosure. In light of the pandemic, and in the interests of safety (i.e., safely accessing accommodations), research reporting suggests that there are some persons who will need to disclose their lived experience [52]. It is critical that such disclosure of neurodivergence can be confidently made in safe and supportive work environments. Furthermore, that any reasonable accommodations needed are expeditiously approved and attained.

\subsection{COVID-related challenges and opportunities for advancing diversity and inclusion (and in particular, including neurodivergence) in the workplace}

Pandemic related issues hold capacity to diminish or enhance the acceptance of neurodivergent staff. Such acceptance might be threatened by reductions in mental wellbeing and associated instances of neuro-discrimination. The U.S. Mental Health Index for workers reports that the risk of general anxiety and depression for males are up by 55 and 69 percent respectively, while fears of redundancy can stop neurodivergent employees from discussing their depression with team members $[28,53]$. Acceptance in the workplace should not be premised upon one being neurotypical or appearing to be neurotypical. Employees should able to safely discuss issues such as anxiety or depression without experiencing judgement or rejection from management or colleagues.

Reflecting a workplace culture that is accepting of neurodivergence, neurodivergent staff should not feel 
threatened in any way about discussing the elevated mental health challenges that some might experience during a pandemic. However, news reports suggest that economic disruptions following the pandemic can translate into autistic persons often being the first to be fired and the last employed [26]. Care is therefore needed so that COVID-related economic challenges do not transform into the unethical practices of 'moving on' neurodivergent employees from the workforce or discriminating against these persons in recruitment processes. These bigoted, neuro-discriminatory practices have no place in ethical workplace cultures that endeavour to be inclusive of neurodivergence, either during a pandemic or at any other time.

Economic and mental health challenges that accompany the pandemic need not necessarily lessen the level of acceptance of neurodivergence in the workplace. Despite COVID-19 related closures to training, the MetLife company continued to enhance its diversity via its recruitment and retention strategies [54]. Moreover, scholarly articles assert that COVID-19 disruptions offer an opportunity to establish new benchmarks for workplace inclusion [20, 27, 55]. Moreover, news reporting raises potential economic gains to be had from the acceptance and recognition of neurodivergence in leadership teams, particularly in troubling times. The Ultranauts organisation managed to continue to grow its revenue (up 50 percent) during COVID-19 with autistic persons in the leadership team [56]. Good leaders view diversity in its many forms, and this outlook encompasses neurodivergence (i.e., diversity of thinking) [57].

According to news reports, aspects of the pandemic hold potential to discourage or encourage empathy for neurodivergent staff. Commencing with indifference, the costs that are imposed by COVID-19 risk seeing a dilution or eradication of diversity and inclusion from company agendas [38]. The pandemic has shown that some organisations see diversity and inclusion as a nicety rather than a central value [38]. Neilson [28] also reports that empathy should not rely on a pandemic that concerns us all. Nevertheless, Miller [58] suggests that communications with staff about the adjustments that can assist them during COVID-19 might encourage empathy more broadly. COVID-19 lockdowns and the fears that are experienced in public places can give neurotypical persons a taste of this discomfort [40, 59]. Indeed, many neurotypical persons have experienced mental health issues under lockdown conditions [6]. Carr-Fanning
$(6$, p.20) in a scholarly article expands upon this matter as follows:

Anecdotal evidence suggests that the COVID-19 lockdown highlighted some of the issues people with different interactional preferences experience trying to function in a world not designed to include them. When lockdown happened, many neurotypical people struggled with their mental health and wellbeing.

Neilson [28] recognises that neurodivergent persons have developed strategies that are useful in trying times and that these can be shared with persons who are having their first lived experiences with anxiety or depression. COVID-19 also holds potential to encourage self-empathy among neurodivergent staff. In this way, the "new normal" together with inclusive organisational cultures might allow neurodivergent staff to be their true selves and to use their abilities to the benefit of their employers $(31,47$, n.p.).

As suggested by news reports, COVID-19 promotes a need for receptive systems and initiatives that are supportive of individual difference, including that of neurological difference [32,60]. Encompassed among these progressive initiatives are Employee Reference Groups (ERGs). ERGs are voluntary groups that are led by employees [61]. These groups aim to help to make workplaces more inclusive by assisting in the understanding of the needs of diverse cohorts $[38,61]$. From a mental health perspective, an ERG can support the mental wellbeing of employees [62]. This review also includes an example whereby neurodivergence has been included among the diversity groups as offered by a workplace [63]. In the wake of COVID, and recognising opportunities to achieve positive change through a greater inclusion, workplaces need to ensure that neurodivergence is not overlooked among the ERGs that are availed to staff.

\subsection{COVID-related guide to accommodating and including neurodivergence in the workplace}

Box 1 provides a baseline guide to supporting neurodivergent employees and prospective employees during (and after) a pandemic. Workplaces that wish to do more than to talk about diversity and inclusion (and in particular, the inclusion of neurodivergent persons) need to invest in these measures. 
Box 1. COVID-related guide to accommodating and including neurodivergence in the workplace.

1. Do not bully neurodivergent staff by micromanaging their work at home activities.

2. Recognise that some neurodivergent persons are at risk of overworking at home.

3. Acknowledge the prospects of remote working to contribute to increased productivity and reduced stress.

4. Ensure that pandemic promoted remote working continues to be available to neurodivergent staff wherever desired and where roles reasonably allow.

5. Reject popular myths about neurodivergent persons (e.g., these persons not requiring social contact) and be accepting of neurodivergence in the workplace.

6. Provide neurodivergent persons with the option of attending virtual social events.

7. Ensure that remote access is available for all meetings and conferences.

8. Recognise that some neurodivergent employees might experience Zoom fatigue.

9. Provide accommodations around Zoom meetings including: one-on-one or group sessions; offering the recordings of meetings; making the agenda available in advance, and providing alternatives to attending live, online meetings (allow contributions to be provided beforehand).

10. Encourage the recruitment, retainment and advancement of neurodivergent staff by availing alternatives to face-to-face interviews (e.g., offer video interviews) and face-to-face communications (e.g., provide text-based communication tools).

11. Include neurodivergence in workplace recruitment, retention and advancement policies.

12. Understand the economic benefits of including neurodivergent persons in leadership teams and recognise the potential for these persons to perform in stressful times (e.g., pandemics).

13. Invest in workplace programs that support an increased acceptance of neurodivergence in leadership teams.

14. Embrace a COVID-19 inspired 'new normal' and develop and maintain a culture that allows neurodivergent persons to safely bring their authentic selves to work.

15. Ensure that neurodivergence is included among the ERGs that are offered in workplaces.

16. Be aware that emails about COVID-19 can be anxiety-producing for some neurodivergent employees and take actions to safely restrict this stressor where needed.

17. For those neurodivergent employees who might desire to return to onsite offices, offer these persons floorspace design options other than open plan arrangements (i.e., provide quiet spaces).

18. Ensure that diversity and inclusion (incorporating neurodivergence) is included and actioned in organisational agendas.

19. Create an accepting workplace culture where staff (and prospective staff) are safe to openly disclose and to discuss their neurodivergence should they wish to.

20. Allow independent workplace audits to examine: a) whether the on-site accommodations provided to neurodivergent staff remain effective when these persons are remote working; and b) the possible extent to which neurodivergent staff who are now remote working due to COVID-19 lockdowns have had reasonable work from home accommodation requests declined prior to the pandemic.

\section{Limitations}

The author has carefully followed scholarly instructions about how to perform a rapid review. Nonetheless, it is appropriate to acknowledge the advice from Kelly, Moher and Clifford [64] that presently, there is no conventional rules for the conducting or reporting of such a review. Being expeditious in nature, Khangura, Konnyu, Cushman, Grimshaw and Moher [34] note that rapid reviews are typically undertaken in five weeks or less. Given this narrow timeframe, this study does not claim to completely cover the literature that might have been published on the topic in question. The author also recognises that the scholarly articles informing this explorative study were notably less in volume than those of news articles. Of the 50 relevant documents, 17 of these were scholarly in nature (i.e., journal articles, thesis or conference proceedings) and 33 were grey literature (i.e., news articles, reports or guides). Further, of the peer-reviewed academic papers included in this review, study limitations included personally expressed views of the author, as well findings recognising a need for future research investigations [20, 27, 55]. Being informed by a rapid review that is itself purposefully confined to the search term and databases applied, the abovementioned guide should thus be treated as a baseline from which future reviews are welcome to build upon.

\section{Conclusion}

What might a COVID-19 inspired 'new normal' mean for supporting neurodivergence in the workplace? This new normal might mean challenging outdated and bigoted excuses for not accommodating neurodivergent persons. It might mean building flexibility into how work-related activities (e.g., interviews, meetings, conversations) are conducted as well making events (e.g., conferences, large gatherings, social occasions) and on-site office layouts more accessible and neurodivergent-friendly. It might also mean affording additional opportunities to have neurodivergence more firmly accepted in leadership teams. Ultimately, a new normal holds notable potential to advance a greater valuing of neurodivergence 
and rejection of neuro-discrimination in contemporary workplaces.

\section{Conflict of interest}

No potential conflict of interest was reported by the author.

\section{Funding}

There was no funding support for this study. This paper is submitted with the understanding that the author is to receive no fees of any sort.

\section{Supplementary data}

Supplementary Tables 1 and 2 are available from https://dx.doi.org/10.3233/WOR-210811.

\section{References}

[1] Schwam A. Outside the box: neurodiversity and the changing workplace 2020 [Available from: https://www.welcome tothejungle.com/en/articles/neurodiversity-at-work

[2] Gilroy S, Haque L. COVID-19 could make the world of work more inclusive. Especially for those who are neurodiverse. 2020. [Available from https://www.weforum.org/ agenda/2020/05/uncovering-neurodiverse-culture-an-opportunity-for-change/

[3] Walker N. Neurocosmopolitanism. 2014. [Available from https://neurocosmopolitanism.com/

[4] Sheffield TUo. Supporting Neurodiverse Staff Whilst we are Working Differently 2021 [Available from: https://www. sheffield.ac.uk/hr/wellbeing/neurodiversity

[5] Paletta D. Equality, capability and neurodiversity. 2013. [Available from https://repository.upenn.edu/miscellaneous _papers/16/

[6] Carr-Fanning K. The Right to Dignity or Disorder? The Case for Attention Deficit Hyperactivity Diversity. Studies in Arts and Humanities Journal. 2020;6(1):14-29.

[7] Faragher J. Are employers losing out on skills of people with autism? Occupational Health \& Wellbeing. 2018;70(5): 16-7.

[8] Gillespie-Lynch K, Dwyer P, Constantino C, Kapp SK, Hotez E, Riccio A, et al. Can We Broaden the Neurodiversity Movement without Weakening It? Participatory Approaches as a Framework for Cross-disability Alliance Building. Disability Alliances and Allies: Emerald Publishing Limited; 2020.

[9] Hughes JA. Does the heterogeneity of autism undermine the neurodiversity paradigm? Bioethics. 2021;35(1):47-60.

[10] Milton D. Disagreeing over Neurodiversity. Psychologist. 2019;32:8
[11] Armstrong T. Their Strengths. Educational Leadership. 2012.

[12] Donnelly C. What employers need to know about neurodiversity benefits 2020 [Available from: https://www.benefitspro.com/2020/07/22/what-employers-need-to-knowabout-neurodiversity-benefits/?slreturn=20210025011830

[13] Kapp SK, Gillespie-Lynch K, Sherman LE, Hutman T. Deficit, difference, or both? Autism and neurodiversity. Developmental Psychology. 2013;49(1):59.

[14] Rothstein A. Mental disorder or neurodiversity? : JSTOR; 2012. [Available from https://thenewatlantis.com/wp-content/uploads/legacy-pdfs/20120823_TNA36Rothstein.pdf

[15] Russell G. Critiques of the neurodiversity movement. Autistic Community and The Neurodiversity Movement. 2019:287.

[16] Davila J, Shectman A, Anandan R. Making Agile More Inclusive. Software Quality Professional. 2020;23(1): 23-31.

[17] Krzeminska A, Austin RD, Bruyère SM, Hedley D. The advantages and challenges of neurodiversity employment in organizations. Journal of Management \& Organization. 2019;25(4):453-63.

[18] Harris C, van der Vloodt R, Maddocks J. Championing neurodiversity and Emotional Intelligence in the workplace. [Available from https://content.psionline.com/whitepaperchampioning-neurodiversity-and-emotional-intelligencein-the-workplace

[19] Sutherland A. Time to celebrate neurodiversity in the workplace. Occupational Health \& Wellbeing. 2016; 68(11): 11 .

[20] Bierema LL. HRD research and practice after 'The Great COVID-19 Pause': The time is now for bold, critical, research. Human Resource Development International. 2020;23(4):347-60.

[21] Kumar P, Ahmad MI, Singh S. COVID-19: A devastating pandemic. Pharmaceutical Sciences. 2020;26(Covid19):S3-S11.

[22] Zandian H, Sarailoo M, Dargahi S, Gholizadeh H, Vosoughi M, Dargahi A. Evaluation of knowledge and health behavior of university of medical sciences students about the prevention of COVID-19. Work. 2021(Preprint):1-7.

[23] Al Eid NA, Arnout BA, Alqahtani MM, Fadhel FH. The mediating role of religiosity and hope for the effect of self-stigma on psychological well-being among COVID-19 patients. Work. 2021(Preprint):1-17.

[24] Lazarides AL, Belay ES, Anastasio AT, Cook CE, Anakwenze OA. Physician burnout and professional satisfaction in orthopedic surgeons during the COVID-19 Pandemic. Work. 2021;Preprint:1-8.

[25] Koh HK, Geller AC, VanderWeele TJ. Deaths from COVID19. JAMA. 2021;325(2):133-4.

[26] Stanford_Medicine. COVID-19 Q\&A: Dr. Lawrence Fung on Impacts on the Neurodiverse Community 2020 [Available from: https://med.stanford.edu/psychiatry/about/ covid19/neurodiverse.html

[27] Thite M. Digital human resource development: where are we? Where should we go and how do we go there? Human Resource Development International. 2020:1-17.

[28] Neilson K. "Not the same boat": neurodiverse employees open up about COVID 192020 [Available from: https:// www.hrmonline.com.au/covid-19/neurodiverse-employees -covid-19/.

[29] Petrosky-Nadeau N, Valletta RG. An Unemployment Crisis after the Onset of COVID-19. FRBSF Economic Letter. 2020;2020(12):1-5. 
[30] Blustein DL, Guarino PA. Work and unemployment in the time of COVID-19: the existential experience of loss and fear. Journal of Humanistic Psychology. 2020;60(5):702-9.

[31] hybris AG: Covid Knights: Corporate social purpose in the time of COVID-19. News Bites - Private Companies. 2020 Apr 16.

[32] Houston D. Cheshire police ranked among most inclusive employers in UK. The Chronicle. 2020 Dec 31.

[33] HOK. Enabling Choices in a More Inclusive covid-19 -era Workspace Ecosystem 2021 [Available from: https://www. hok.com/ideas/research/enabling-choices-in-a-more-inclusive-covid-19-era-workplace-ecosystem/

[34] Khangura S, Konnyu K, Cushman R, Grimshaw J, Moher D. Evidence summaries: the evolution of a rapid review approach. Systematic Reviews. 2012;1(1):1-9.

[35] Braun V, Clarke V. Using thematic analysis in psychology. Qualitative Research in Psychology. 2006;3(2):77-101.

[36] Sargent K. The Latest Workplace Design Initiative? Neurodiversity 2020 [Available from: https://www.metropolismag.com/architecture/workplace-architecture/workplace-design-neurodiversity/

[37] hybris AG: 3 Management Tips for Topsy Turvy Times. News Bites - Private Companies. 2020 Oct 15.

[38] Will diversity survive after the pandemic?: Marshalling employee support can ensure valuable activity stays on the agenda, writes Maxine Kelly. Financial Times. 2020 Aug 31 .

[39] Beery Z. When the World Shut Down, They Saw It Open a Bit More: [Style Desk]. New York Times. 2020 Aug 27.

[40] Gaunt M. Firms are urged to rethink inclusion. The Yorkshire Post. 2020 May 18.

[41] Hannam-Swain S, Bailey C. Considering Covid-19: Autoethnographic reflections on working practices in a time of crisis by two disabled UK academics. Social Sciences \& Humanities Open. 2021;4(1):100145.

[42] Ishihara D. Belgium: Neurodiversity At Work 2020 [Available from: https://www.mondaq.com/employment-andworkforce-wellbeing/1007384/neurodiversity-at-work

[43] Miller KL. Seeking clarity. The Washington Post. 2020 Oct 14.

[44] Wright B, Spikins P, Pearson H. Should Autism Spectrum Conditions Be Characterised in a More Positive Way in Our Modern World? Medicina (Kaunas). 2020;56(5):233.

[45] Neurodiverse Employees May Need Accommodations for Remote Work. HRNews. 2020.

[46] Terise N, Armstrong A. Supporting neurodivergent staff in the post-lockdown workplace 2020 [Available from: https://www.personneltoday.com/hr/neurodiversitypost-lockdown-coronavirus-advice/.

[47] Moore G. Neuro-diversity is an Iso-Superpower 2020 [Available from: https://www.macquarie.com/au/en/perspectives/neurodiversity-is-an-iso-superpower.html.

[48] CoverMyMeds LLC: Pharmacists: The Often-Overlooked Providers. News Bites - Private Companies. 2020 Dec 10.

[49] Wendel SE Investor Day (virtual) - Final. Fair Disclosure Wire. 2020 Nov 04.

[50] Creased_Puddle. Empowering Neurodiversity in the Workplace during Covid-19 2020 [Available from: https://www. creasedpuddle.co.uk/wp-content/uploads/2020/04/NeuroteamC19-Covid-and-Neurodiversity-Guidance-for-Organisations.pdf.

[51] CoverMyMeds LLC: Generational Gaps in Medication Access. News Bites - Private Companies. 2020 Nov 25.

[52] Clifton S. Hierarchies of power: disability theories and models and their implications for violence against, and abuse, neglect, and exploitation of, people with disability. 2020 Oct 22. [Available from https://apo.org.au/node/309065

[53] National Alliance of Healthcare Purchaser Coalitions: Men's Risk of Depression Up 69\%. Targeted News Service. 2020 Nov 21.

[54] Kyriakou S. MetLife secures provider award. London: The Financial Times Limited; 2020.

[55] Mitsakis FV. Human resource development (HRD) resilience: a new 'success element'of organizational resilience? Human Resource Development International. 2020;23(3):321-8.

[56] MIT: Leveraging the Power of Neurodiversity. Targeted News Service. 2020 Dec 01

[57] Counter R. Canada's 50 Best Executives 2020: They led their companies through COVID-19 and built a better country in the process: The Report on Business Best Executive Awards showcases 50 EVPs, SVPs and members of C-suite. The Globe and Mail (Online). 2020.

[58] Miller KL. Boss considers disclosing autism to employees. The Washington Post. 2020 Jul 05.

[59] Tingley K. How Architecture Could Help Us Adapt to the Pandemic. New York Times (Online). 2020.

[60] Kumar R. Supporting well-being in resource-scarce environments [Blogs]. Blogs (The Times of India Online Edition and The Economic Times Online Edition). 2020 Aug 04.

[61] CoverMyMeds LLC: Putting Wellness First: A Look at our Newest Employee Resource Group. News Bites - Private Companies. 2020 Dec 16.

[62] CoverMyMeds Campus Update: A Sneak Peek At Our Workspace - Part One. News Bites - Private Companies. 2020 Dec 22.

[63] Kilpatrick C. How COVID has changed the lives of women in IP. Managing Intellectual Property. 2020.

[64] Kelly SE, Moher D, Clifford TJ. Quality of conduct and reporting in rapid reviews: an exploration of compliance with PRISMA and AMSTAR guidelines. Systematic Reviews. 2016;5(1):1-19.

[65] Defining the Bionic Business: 5 traits define a new era in employee communication and engagement. M2 Presswire. 2020 Dec 08.

[66] Reed Smith associate named fourth most influential disabled person in the UK. News Bites - Private Companies. 2020 Oct 29.

[67] Bahl S. Commemorating the inclusion of 'Specially-Abled' Business World. 20202020 Dec 03.

[68] Dosser T. K.E.E.N. continues important work during pandemic. Sherwood Park News (Online). 2020.

[69] PattiWaldmeir. Overlooked workers tapped in challenging times. Financial Times. 2020 Mar 18.

[70] Walling M. Flexibility and change: How the intellectual disability community is facing COVID-19. TCA Regional News. 2020 Jul 23.

[71] Waites B, Breslin G, Bell N, Thomson L, Fraser J, Mackay $\mathrm{G}$, et al., editors. Covid-related anxiety and distress in the workplace: A guide for employers and employees: Covid and anxiety in the workplace2020: British Psychological Society.

[72] Magni S. Virtual Theatre Of Neurodiversity: A Workbook for Educators, Facilitators and Performers with Disabilities: Faculty of Environmental Studies in partial fulfillment of the requirements ...;2020.

[73] NeurodiversityHub. Resources for Virtual Study and Work 2021 [Available from: https:www.neurodiversityhub. org/covid19]. 